\title{
Analysis of EEG activity during sleep - brain hemisphere symmetry of two classes of sleep spindles
}

\author{
Faculty of Physics and Applied Computer Science, AGH University of Science and Technology, \\ 30 Mickiewicza Ave., 30-059 Krakow, Poland \\ e-mail: smolen@novell.ftj.agh.edu.pl
}

This paper presents automatic analysis of some selected human electroencephalographic patterns during deep sleep using the Matching Pursuit (MP) algorithm.

The periodicity of deep sleep EEG patterns was observed by calculating autocorrelation functions of their percentage contributions. The study confirmed the increasing trend of amplitude-weighted average frequency of sleep spindles from frontal to posterior derivations. The dominant frequencies from the left and the right brain hemisphere were strongly correlated.

Key words: polysomnography, delta waves, sleep spindles, Matching Pursuit, adaptive approximations.

\section{Introduction}

Polysomnographic recording is a complementary diagnostic procedure which yields principal clinical and cognitive information about the human brain activity. The sleep EEG patterns may change under the influence of degenerative diseases. Abnormal polysomnograms are successfully used in insomnia treatment and in the diagnosis of illnesses, such as epilepsy, narcolepsy, psychiatric disorders, tumour changes, central nervous system dysfunctions or in the effect of various drugs [5]. Thus, all EEG automatic investigations, which have the purpose of assisting physicians in their visual inspections, are greatly desirable. 
Mathematical methods of neuron bioelectrical activity processing, especially polysomnographic signal, are based mainly on time-frequency analysis. The reason for this is that these approximations provide an optimal tool to produce various types of non-stationary signal representations. This approach is useful when there is a need to decompose the signal in detail and identify its most significant components, i.e. both long- and short-time ones, in the same framework [7]. Many of the earlier results showed good compatibility of signal adaptive time-frequency approximations with their visual detections [3]. This is the reason why our approach uses the Matching Pursuit (MP) algorithm with the time-frequency dictionaries.

This paper takes into account basic waves and structures which occur periodically in polysomnographic recordings during deep sleep, such as delta waves and sleep spindles (SS), the most intriguing polysomnographic pattern. SS are rhythmic transients present during non-rapid eye movement (non-REM) sleep. Their spectra concentrate in the range of $11-15 \mathrm{~Hz}$, and the amplitudes mostly do not exceed $50 \mu \mathrm{V}$ in adult humans. Many early analyses proved the existence of two types of sleep spindles, differentiated on the basis of their frequency and topographical location: low frequency spindles (LFS) more pronounced in frontal derivations with frequency spectrum peak at roughly $12 \mathrm{~Hz}$, and high frequency spindles (HFS) predominant in posterior derivations around $14 \mathrm{~Hz}$ $[6,7]$. Results of two distinct spindle components have also been confirmed more precisely in brain electrical activity estimation by means of low-resolution electromagnetic tomography (LORETA) [1]. Two areas in the brain were identified as main sources of cortical electrical spindle activity: Brodmann areas 9 and 10 (in frontal cortex) for LFS, and Brodmann area 7 (in precuneus) for HFS. These regions have connections with the dorsal thalamus, where sleep spindles are generated.

In this study, several observations of the specific EEG pattern have led to important conclusions about the following tasks:

- sleep spindles topography,

- sleep spindles correlation between the right and the left brain hemisphere,

- percentage contribution and periodicity of sleep waves from deep sleep.

\section{Material}

Polysomnographic recordings of 5 healthy volunteers (two females and three males, aged 20-57 years, mean age 33 years) were considered in the study. None of these 
subjects had a history of sleep disturbances. Data were acquired from 15 selected channels according to the 10-20 system, two mastoid electrodes in reference and a ground electrode placed between Fp1 and Fp2. The Silver/Silver Chloride surface electrodes were applied with Electrode Cream EC2. The maximum value of tolerable resistance was $20 \mathrm{k} \Omega$. An EEG amplifier contained a 16 bit analog-to-digital converter. Parallel sampling for all channels was used, therefore there was no problem of phase shifts between channels. Sampling frequency was $500 \mathrm{~Hz}$.

After data collection, the signal was filtered with a bandpass filter $(0.5-30 \mathrm{~Hz})$ and a filter excluding the frequency of $50 \mathrm{~Hz}$. From the signal of each channel its mean and the mean of signals from $\mathrm{A} 1$ and $\mathrm{A} 2$ derivations were subtracted. Then quadruple resampling to $125 \mathrm{~Hz}$ was applied.

\section{Methods}

\section{Matching Pursuit algorithm}

The iterative method of Matching Pursuit introduced by Mallat and Zhang in 1993 [4] is based on adaptive description of the tested signal by means of a huge function dictionary $G=\left\{g_{1}, g_{2}, \ldots, g_{n}\right\}$, where $\left\|g_{i}\right\|=1$. At first, the waveform $g_{\gamma 0}$ which creates a maximal scalar product with the signal, is selected from the dictionary. Thus the fitting to the signal is most significant. In each successive step, the analytic function $g_{\gamma n}$ (pattern) is made running along the analyzed signal $R^{n} x$, yielding as a result a correlation function and a residual function. The best matching pattern and the corresponding time distribution of pattern-signal likelihood are adopted as decomposition coefficients, while the residual function is subject for further decomposition. After $n$ steps of decomposition, the signal can be expressed as a convolution of $n$ analytic signals weighted by the (time-pattern) decomposition coefficients and the residual signal not sufficiently well fitting to any of the dictionary component. These operations are presented below by the following set of equations [3]:

$$
\begin{aligned}
R^{0} x & =x \\
R^{n} x & =\left\langle R^{n} x, g_{\gamma_{n}}\right\rangle g_{\gamma_{n}}+R^{n+1} x \\
g_{\gamma_{n}} & =\arg \max _{g_{i} \in G}\left|\left\langle R^{n} x, g_{i}\right\rangle\right|
\end{aligned}
$$


When the particular time-frequency waveforms (atoms) have been fitted to the analyzed signal, the procedure is convergent to $x$ :

$$
x=\sum_{n=0}^{\infty}\left\langle R^{n} x, g_{\gamma_{n}}\right\rangle g_{\gamma_{n}}
$$

A redundant dictionary from which the functions are chosen consists of waveforms $g_{\gamma}(t)$. They are generated by translating $(u)$, scaling $(s)$ and modulating the window function $g(t)$ :

$$
g_{\gamma}(t)=\frac{1}{\sqrt{s}} g\left(\frac{t-u}{s}\right) e^{i 2 \pi f t}
$$

Since Gabor function has an optimal localization in the time-frequency space, it is perfectly suitable for a dictionary construction. In most applications, the signal $x(t)$ is a real function and then it is defined as:

$$
g_{\gamma}(t)=K(\gamma, \varphi) e^{-\pi\left(\frac{t-u}{s}\right)^{2}} \sin (2 \pi f(t-u)+\varphi)
$$

where: $K(\gamma, \varphi)$ is a normalizing factor such that $\|g(\gamma, \varphi)\|=1$, and $\varphi$ is a phase.

In high-resolution time-frequency studies the Matching Pursuit is undoubtedly highly predominant over other methods. Sleep EEG recordings have the structures which differ in their time span, frequency and shape. There appear both long rhythmical components and transients with instantaneous frequency, so the use of MP and Gabor functions for the analysis is clearly justified.

\section{EEG structure parameterization}

Signals from each of the fifteen channels were decomposed separately into stochastic time-frequency dictionaries of Gabor functions in epochs of 20 s. To that aim, free MP4 software (available from http://eeg.pl/mp) was used. The application was written by Ircha under the supervision of Durka at the Laboratory of Medical Physics at Warsaw University in Poland [2].

Parameters describing the fitted waveforms were selected from the following variables of Gabor function: frequency, time width, exact time position of occurrence 
and peak-to-peak amplitude. After the decomposition of signals to time-frequency atoms with the accepted signal energy explanation of 95\%, proper interval limits were set on the matched waveforms to choose the desirable pattern of selected sleep structures. Exact threshold criteria for the corresponding structures are given in Table 1. Following Durka [2], the frequency range of sleep spindles was fixed slightly wider than the classical range which is between 12 and $14 \mathrm{~Hz}$.

Table 1. Values of basic parameters where the structures corresponded to delta waves and sleep spindles

\begin{tabular}{|l|c|c|c||}
\cline { 2 - 4 } \multicolumn{1}{c|}{} & Frequency $\mathrm{f}[\mathrm{Hz}]$ & Amplitude $\mathrm{A}[\mu \mathrm{V}]$ & Time width $[\mathrm{s}]$ \\
\hline \hline Delta waves & $0.5 \leq \mathrm{f} \leq 4$ & $\mathrm{~A} \geq 75$ & $\mathrm{~S} \geq 0.5$ \\
\hline Sleep spindles & $11 \leq \mathrm{f} \leq 15$ & $\mathrm{~A} \geq 15$ & $0.5 \leq \mathrm{S} \leq 2.5$ \\
\hline
\end{tabular}

A parametric description of the signal is a very convenient starting point for further investigations. Such a decomposition and complete dictionary of Gabor functions make it possible to inspect signal properties and establish many interesting relationships between them. A large number of spatial distributions and dependencies can easily be made up.

\section{Percentage contributions and their autocorrelation functions}

The first observation in our study involved calculating the percentage contribution of some selected EEG structures, such as delta waves and sleep spindles from the deep sleep stadium of a nightlong sleep. Each sample of the signal in a given time period of $20 \mathrm{~s}$ was verified if it was included in the time range of any atoms corresponding to slow waves or sleep spindles. Then all samples which satisfied this criterion were totalized and the percentage contributions depending on time were prepared. Autocorrelation functions of calculated contributions were obtained from the following formula:

$$
R_{x x}(k)=\frac{1}{N-|k|} \cdot \frac{1}{\sigma^{2}} \sum_{n=0}^{N-|k|}\left(x(n)-x_{\text {mean }}\right)\left(x(n-k)-x_{\text {mean }}\right)
$$

where: $k$ is the time shift in samples, $N$ is the number of all signal samples, $x_{\text {mean }}$ is the signal mean value, and $\sigma$ is the standard deviation of the analyzed signal. 


\section{Topography of sleep spindles}

In order to study the space of sleep spindle appearance in which each type of spindles occurs and to verify previous scientific studies, topographical representations of their amplitudes (Figure 1) and power (Figure 2) in the function of frequency were determined. These figures consist of fifteen plots corresponding to fifteen selected EEG derivations. A single sleep spindle is equivalent to one point.
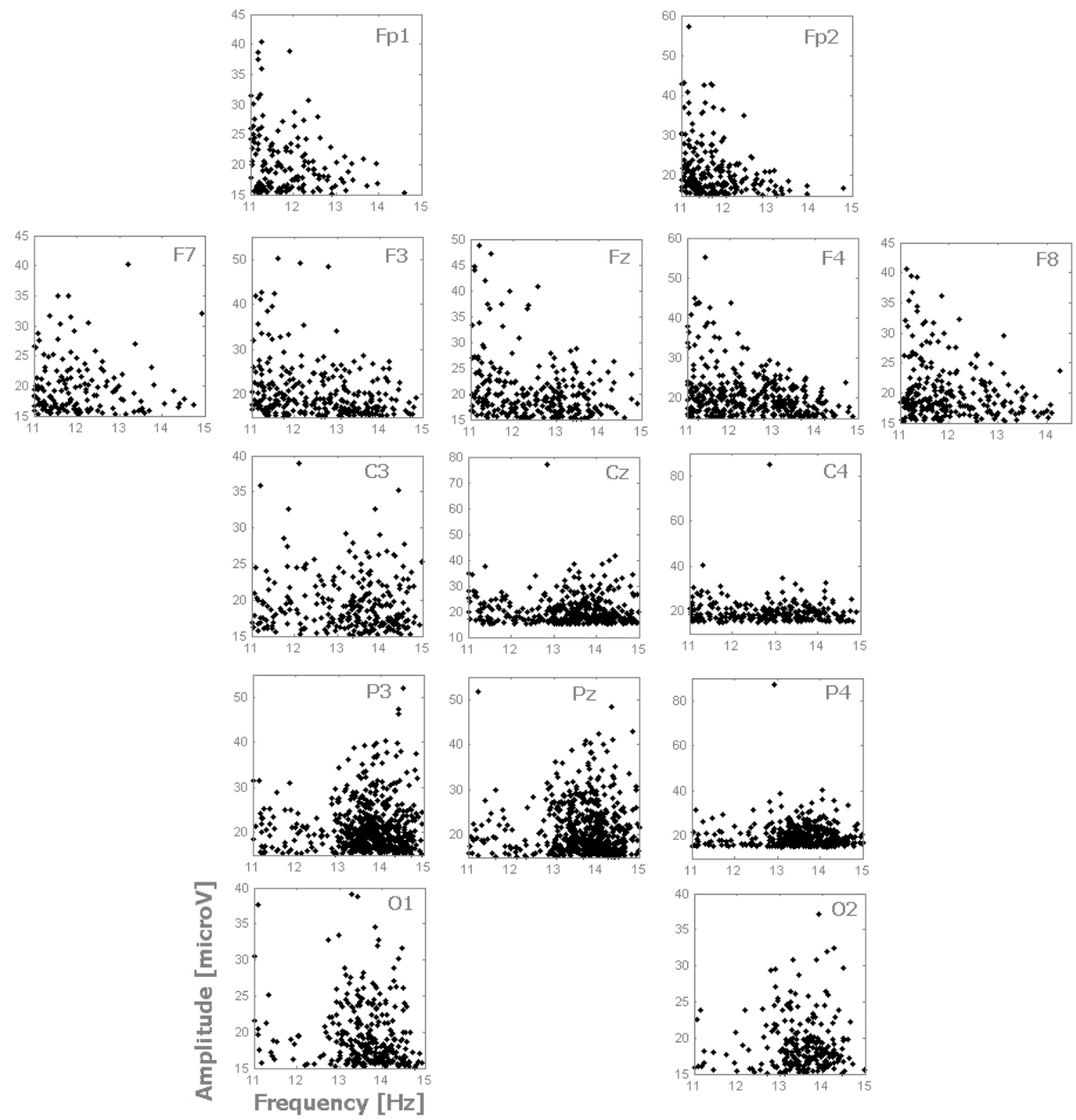

Figure 1. Topographical representation of sleep spindle amplitude in the function of frequency. Fifteen plots correspond to fifteen different chosen derivations. A single sleep spindle is equivalent to one point 
Sleep spindle frequency band of $11-15 \mathrm{~Hz}$ was divided into 40 diminutive intervals of $0.1 \mathrm{~Hz}$ width. For each of them, the power spectra were calculated with the use of formula (6) and afterwards approximately fitted curves were shown in Figure 2.

$$
P_{k}=\sum_{i}^{N} A_{i}^{2}
$$

where: $k=1,2, \ldots, 40$ (number of intervals), $A_{i}$ is the amplitude of the single sleep spindle, and $N$ is the number of sleep spindles which belong to the given interval

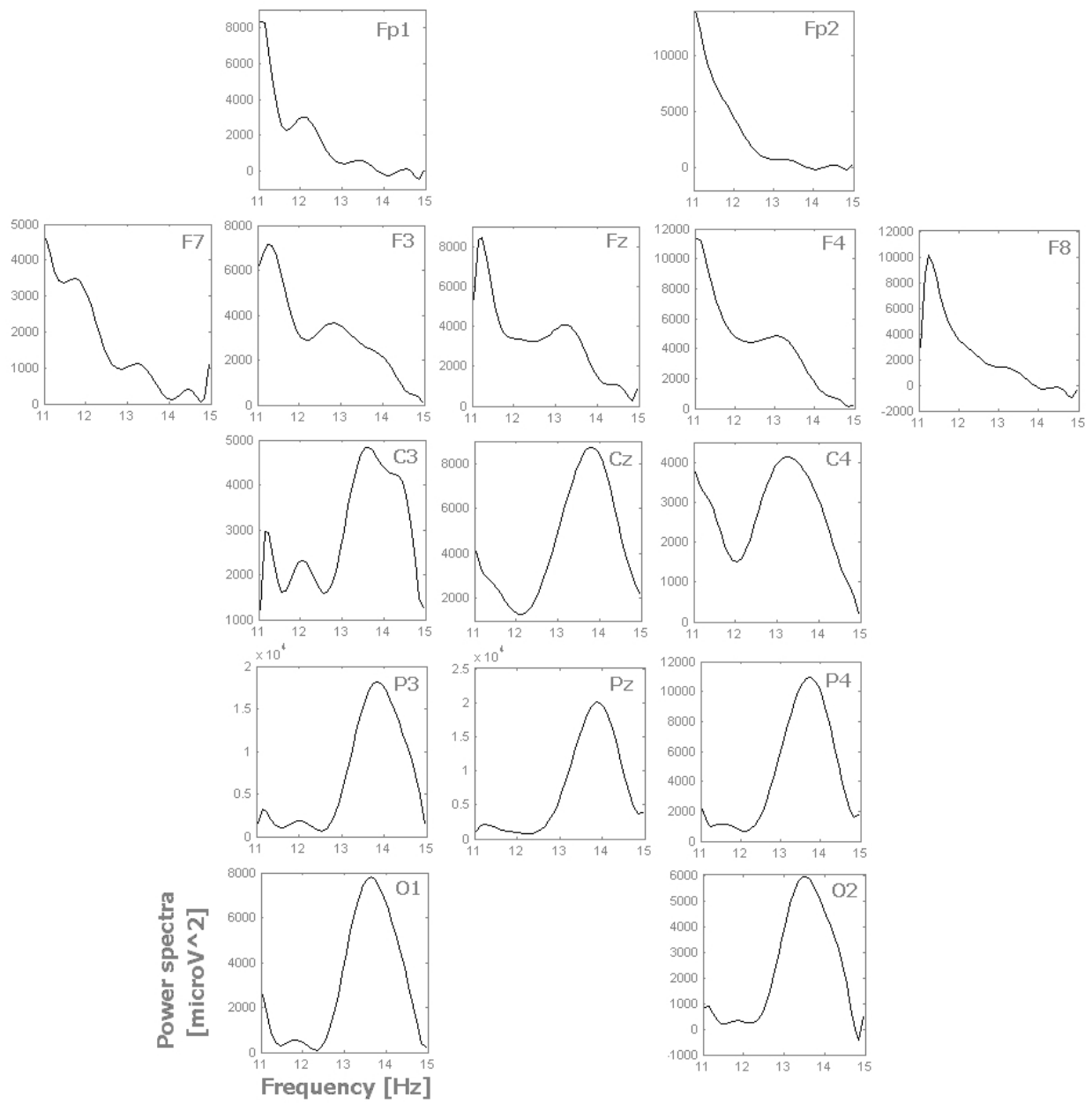

Figure 2. Topographical distribution of sleep spindle power spectra depending on frequency. Fitted curves of sleep spindle power at a given frequency 


\section{Results}

\section{Percentage contribution of} delta waves and sleep spindles

Figures 3 and 4 present the contributions and their autocorrelation functions for all the studied subjects from $\mathrm{Cz}$ channel appropriately for sleep spindles and delta waves. It can be seen that both slow waves and sleep spindles repeated periodically during the sleep. This periodicity is quite disturbed by other frequency components, since the stages and phases of physiological sleep can iterate but in a different order. Observation of the time evolution of delta waves suggests that there is a decrease in their contribution towards the end of the sleep. This is the time when the human body rests and regenerates.

\section{Topography of sleep spindles}

It can be seen (Figure 1 and Figure 2) that low- and high-frequency spindles predominate in various
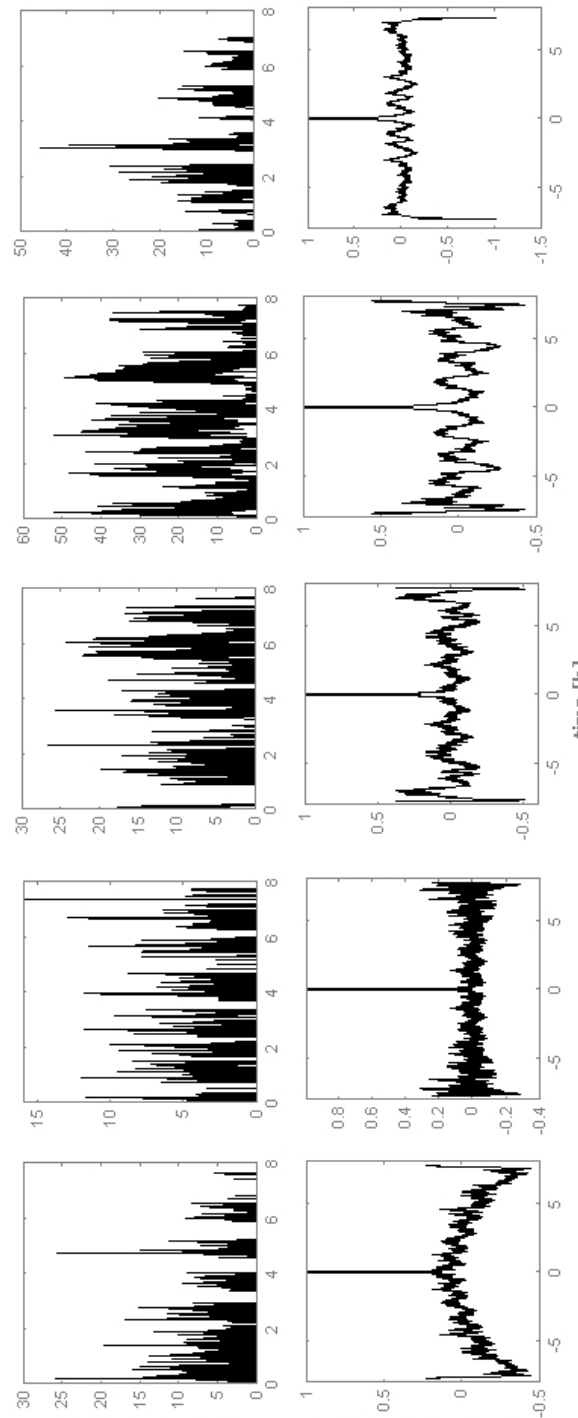

SS to abequaว.dad

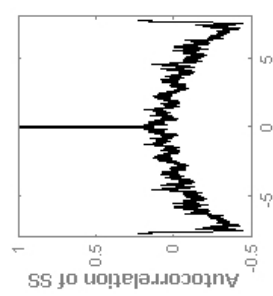

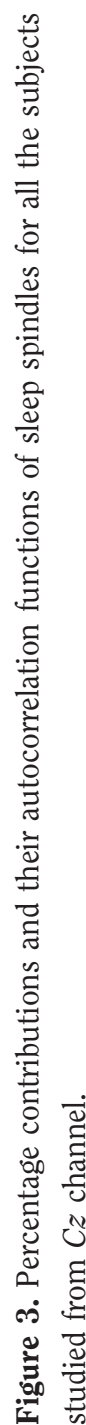
regions of the brain. Frontal electrodes registered a larger number of LFS in contrast to parietal electrodes where HFS predominate. These two pictures were extracted for one typical volunteer, but other subjects revealed similar relationships. 

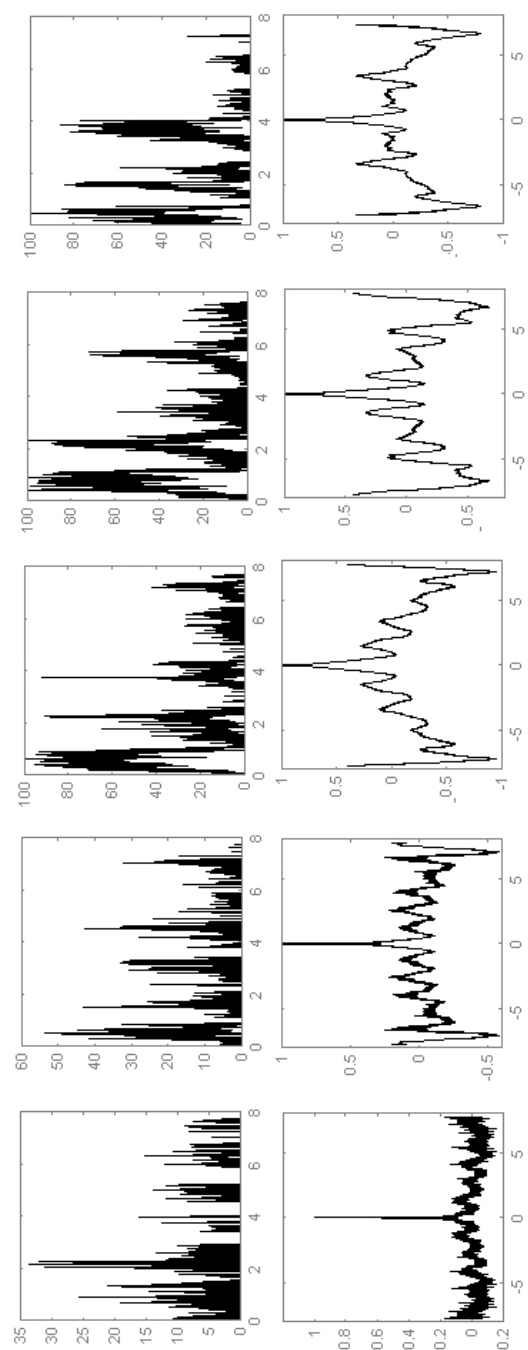

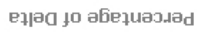

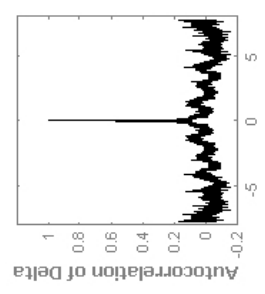

The above mentioned results encouraged us to test the phenomenon of spindle frequency distinctions more precisely. For all the subjects and EEG electrodes, the weighted means of spindle frequency were calculated. Naturally, the weights in this case were amplitudes of the structures. The increasing tendency of the average frequency was observed from the frontal in the direction of posterior derivations $(F p \rightarrow F \rightarrow C$ $\rightarrow P$ ) for each brain hemisphere in all the investigated volunteers. Moreover, the growth tended to be a linear function, whereas sleep spindle average frequency from occipital electrodes $(O)$ had nearly the same or slightly smaller values than in parietal derivations $(P)$.

The final task in our research was to study the brain hemisphere symmetry of both classes of sleep spindles. For that reason, previously prepared regression lines of spindle average frequency growth towards the posterior electrodes were tested. The hypothesis that frequency dependence is the same in the left as in the right hemisphere was confirmed since no statistically significant difference was found between the corresponding regression lines. The signals from occipital electrodes, which were not considered in this analysis as not fitting to the linear regression line of frequency increase, had also very close average frequency values in both hemispheres. 


\section{Discussion}

In this study, we have proposed the automatic analysis of the human polysomnographic activity during nightlong sleep. The method of Matching Pursuit provides a complete description of the sleep EEG patterns. These patterns consist of different frequency waves and other specific structures. The most important fact when using the MP is a possibility of providing a description of EEG structures depending on their frequency, amplitude, occurrence and duration in time. The obtained signal description can lead to various significant conclusions.

In accordance with the expectations, the percentage contributions of structures from the deep sleep stadium repeated periodically during overnight sleep and the contribution of delta waves decreased with sleep time. The correspondence of the scalp region and the dominant frequency was also observed in our research. Moreover, amplitude-weighted average frequencies of sleep spindles had an increasing tendency from the frontal to the posterior electrodes, and the values of the dominant frequency from the left and the right brain hemisphere were directly correlated.

\section{Conclusions}

A Matching Pursuit method demonstrated its ability of providing a detailed decomposition of an EEG pattern, especially polysomnographic recordings. The main advantage of this method is its high resolution in the time-frequency domain. Our research confirmed some previous analysis concerning the contribution, periodicity and topography of specific EEG patterns. Therefore, we are now preparing new detailed inspection of sleep spindle mean frequency symmetry of both brain hemispheres. Our results have stimulated us to undertake further study of the correspondence between LFS and HFS sleep spindle topography so as to obtain a better understanding of the neurophysiological mechanisms of sleep spindles. 


\section{Acknowledgements}

All MP4 calculations in this research were carried out with the IBM BladeCenter HS21 cluster (mars) at the Academic Computer Centre CYFRONET AGH. This research was supported by grant No. MNiSW/IBM_BC_HS21/AGH/035/2008.

\section{References}

[1] Anderer P, Klosch G, Gruber G, Trenker E, Pascual-Marqui RD, Zeitlhofer J., Barbanoj MJ, Rappelsberger P, Saletu B. Low-resolution brain electromagnetic tomography revealed simultaneously active frontal and parietal sleep spindle sources in the human cortex. Neurosci. 2001; 103(3): 581-592.

[2] Durka PJ, Malinowska U, Szelenberger W, Wakarow A, Blinowska KJ. High resolution parametric description of slow waves sleep. J Neurosci Methods. 2005; 147: 15-21.

[3] Malinowska U, Durka PJ, Blinowska KJ, Szelenberger W, Wakarow A. Micro- and macrostructure of sleep EEG. IEEE Eng Med Biol Magazine. 2006; 25: 26-31.

[4] Mallat S, Zhang Z. Matching Pursuit with time-frequency dictionaries. IEEE Trans Signal Process. 1993; 41: 3397-3415.

[5] Traczyk WZ, Trzebski A. [Human Physiology with Elements of Applied and Clinical Physiology]. Warsaw: PZWL; 1989. Vol. 1. Polish.

[6] Ventouras EM, Alevizos I, Ktonas PY, Tsekou H, Paparrigopoulos T, Kalatzis I, Soldatos CR, Nikiforidis G. Independent components of sleep spindles. Conf Proc IEEE Eng Med Biol Soc. 2007; 1: 4002-4005.

[7] Zygierewicz J, Blinowska KJ, Durka PJ, Szelenberger W, Niemewicz Sz, Androsiuk W. High resolution study of sleep spindles. Clin Neurophysiol. 1999; 110: 2136-2147. 
